\title{
Structure Optimization of UHV RIP Oil-SF6 bushings Based on Improved Equal Margin Design Method and Back-Propagation Neural Network
}

\author{
Zhang Shiling ${ }^{1}{ }^{*}$ \\ ${ }^{1}$ State Grid Chongqing Electric Power Company Chongqing Electric Power Research Institute, Chongqing, 401123
}

\begin{abstract}
Equal margin design method based on the classic analytic formula is widely used in development of extra-high voltage bushing products, and its effectiveness and practicality have been fully validated. However, model and temperature factors have significant impact on internal E-field distribution of UHVAC and UHVDC bushing condenser, which traditional analytic formula is difficult to evaluate quantitatively, so it's necessary to improve traditional equal margin design method. Firstly, basic principles of equal margin design method and its software package were briefly described, and the laws of model and temperature factors influencing on condenser E-field were investigated on FEM (finite element method) computing platform. Based on these, mathematical model of improved equal margin design method for bushing condenser was established, and flow chart of optimization process combining FEM electro-thermal coupling calculation with genetic algorithm was presented. The improved method was applied to design of UHV RIP oil-gas prototype to realize uniform axial E-field distribution along bushing condenser and equal partial discharge margin between adjacent foils. Bushing condenser was fabricated according to above optimized design structure, and has passed all type tests. In the paper, the FEM electro-thermal coupling calculation method was applied to the inner insulation optimization design to make bushing condenser's design more suitable. The paper can provide some theoretical guidelines for research and development of other bushings in UHV level.
\end{abstract}

\section{Introduction}

Capacitive bushing is a common type of bushing in $\mathrm{UHV} / \mathrm{UHV}$ power system at present: a capacitor core is added between the central guide rod and the flange as the internal insulation, and there are multi-layer metal plates in the capacitor core to control the electric field uniformity inside and on the surface of the bushing. The inner insulation of the capacitor bushing, i.e. capacitor core, is the key component in the electrical design and calculation. The design of capacitor core should follow the following principles: (1) no harmful partial discharge under the maximum working voltage; (2) no axial flashover at the end of bushing under the rated power frequency breakdown voltage (1.2 times dry withstand voltage). The traditional design method of equal step and equal capacitance is simple in principle, but the overall electrical performance of the bushing core needs to be improved due to the uneven axial field strength. In the $1980 \mathrm{~s}$, in order to reduce the structure size of bushing core, a design method of equal thickness and unequal step was proposed. The key is to determine the step length of each layer of electrode plate to make the axial field strength as uniform as possible, but the adjustment process is rather complicated. In the 1990s, new design method of capacitor core, equal margin design method was proposed in reference[1]. It was proved theoretically that this method can effectively improve the initial partial discharge voltage of bushing core compared with the traditional method, and the theoretical results were verified by experiments. Furthermore, in reference[2], the equal margin method is developed as software package for the optimal design of capacitor core of high voltage capacitive bushing. The software package has been used up to now, and has successfully designed capacitive bushing products under various voltage levels. At present, the rapid development of AC/DC UHV transmission in China puts forward higher requirements for the design and development of UHV capacitive bushing. Therefore, it is an urgent problem to be solved whether the current design software package of bushing capacitor core needs to be further improved under UHV voltage level.

It is necessary to improve the design program of bushing capacitance core in following two aspects: (1) calculate the radial and axial field strength distribution inside bushing core as accurately as possible; (2)couple the temperature field distribution of the core into the electric field design. The capacitance core is the main capacitance part of the bushing, and the radial and axial electric field distribution is mainly affected by the size

*zhangshiling@cq.sgcc.com.cn 
and relative position of the electrode plate, but the stray capacitance will also be introduced into the metal parts such as the bushing center guide rod, the connecting sleeve and the equalizing ball, which will affect the internal electric field distribution of the core[3-6]. On the other hand, under the rated operation conditions of the $\mathrm{AC} / \mathrm{DC}$ bushing, the temperature gradient inside and outside the core, the radial and axial electric field distribution will be established. The traditional optimization design software package of bushing capacitor core can not take the above two factors into account in the design process. Now these two factors are referred to as model influence factor and temperature influence factor respectively. On the basis of the traditional analytical design of the existing software package, calculation method of electro-thermal coupling field should be found.

In engineering practice, it is usually very difficult to get the exact analytical solution of the problem. According to the specific situation, the boundary and initial conditions can be given, and the numerical solution can be obtained by numerical method. The finite element method is one of the most effective and widely used numerical calculation methods. Now the advent of large capacity computer also lays the foundation for the high-precision and efficient electromagnetic numerical calculation. Taking the design of high-voltage capacitive bushing core as an example, the classical analytical formula can be deduced to calculate the field strength value accurately and complete the design of electrode plate size and relative position when bushing core is considered alone. However, if the model and temperature are considered, it is difficult to obtain the analytical expression of the internal electric field of the bushing core. In view of this, this paper uses the finite element numerical calculation method to obtain the full thickness of the bushing. The core structure optimization process based on the finite element electro-thermal coupling calculation and the genetic algorithm is introduced. Considering the influence factors of temperature and model, basic requirements of bushing core design are realized: the radial field strength meets the control requirements, and the axial field strength and partial discharge margin between plates are evenly distributed. The improved equal margin design method is applied to the optimization design of inner insulation of $\mathrm{AC}$ $1100 \mathrm{kV}$ dry oil and gas bushing core, and the prototype has passed all type tests.

\section{Equal margin design method of traditional bushing core and its software package implementation}

\subsection{The design method of the traditional bushing capacitor core equal margin}
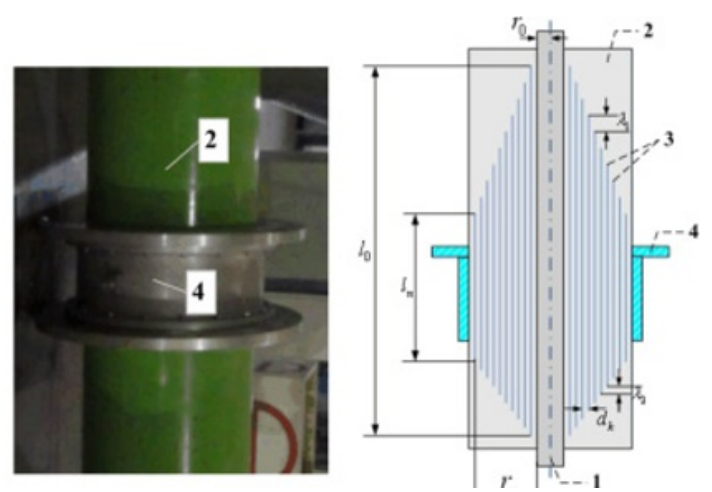

$\begin{array}{ll}\text { (a) Core material object } & \text { (b) Layout of inner plate of core }\end{array}$

Fig.1 Photo of bushing condenser and foil arrangement

The layout of the bushing capacitor core and its internal plate and the characterization of relevant parameters are shown in Figure 1. Assuming that the bushing capacitor core contains $n$ layers of plates, the initial voltage of PD between layers can be expressed by (1)[7-9]:

$$
\Delta U_{i}=K d^{0.5}
$$

Where $K$ is the coefficient, for oil paper bushing, the harmful partial discharge coefficient can be 4.5 , and the flash-over discharge coefficient can be 13.5. For the epoxy impregnated paper dry bushing, its value needs to be further studied and determined. $d$ is the thickness of insulating layer between two plates $(\mathrm{mm}) . \Delta U_{i}$ is the initial partial discharge voltage $(\mathrm{kV})$ of insulating layer. If the design method of equal capacitance is adopted, the initial partial discharge voltage of the whole bushing core is:

$$
U_{i}=n K d_{\min }^{0.5}
$$

In the above formula, $d_{\text {min }}$ is the minimum insulation thickness between plates $(\mathrm{mm}) . U_{i}$ is the initial partial discharge voltage of bushing $(\mathrm{kV})$. If the number of internal layers $n$, the distribution of axial electric field $E_{a}$ and the overall size of the core remain unchanged, and the insulation thickness between the layers is properly adjusted, the inter layer margin $\Delta U_{i} / \Delta U$ can be equal.

At this time, the initial partial discharge voltage of bushing is:

$$
U_{i}^{\prime}=\sum_{k=1}^{n} \Delta U_{i k}=K \sum_{k=1}^{n} d_{k}^{0.5}
$$

Among them, $U_{i}^{\prime}$ is the initial partial discharge voltage of bushing core in the case of equal margin design. Compared with formula $(2) \sim(3)$, it can be seen that the initial voltage of partial discharge is higher than $U_{i}$, so equal margin design can effectively improve the initial voltage of partial discharge. The equal margin 
method can be carried out on the basis of equal step and equal capacitance design. In fact, the distribution of the radial electric field is independent of the thickness $D_{\mathrm{K}}$ of the insulation layer between the plates[10,11]. Therefore, it can be assumed that the radial electric field $E_{r k}$ at each plate is approximately equal to the radial electric field strength under the design of equal step and equal capacitance. In equal margin design, the ratio of the initial partial discharge voltage of each insulation layer to the voltage distributed on the layer should be equal to a constant:

$$
\frac{\Delta U_{i k}}{\Delta U_{k}}=\frac{E_{i k}}{E_{r k}}=\frac{K\left(d_{k}\right)^{-0.5}}{E_{r k}}=A
$$

It is obtained that the insulation thickness $d_{k}$ of layer $k$ is:

$$
d_{k}=\left(\frac{A E_{r k}}{K}\right)^{-\frac{1}{0.5}}
$$

And because:

$$
r_{n}-r_{0}=\sum_{k=1}^{n} d_{k}=\sum_{k=1}^{n}\left(\frac{A E_{r k}}{K}\right)^{-\frac{1}{0.5}}
$$

According to this, we can get the following conclusions:

$$
A=\frac{K\left(\sum_{k=1}^{n} E_{r k}^{-\frac{1}{0.5}}\right)^{0.5}}{\left(r_{n}-r_{0}\right)^{0.5}}
$$

Thus, the following iterative scheme can be formed, in which the calculated value of the first iteration is expressed by superscript (1):

$$
\begin{gathered}
d_{k}=\left(\frac{A^{(1)} E_{r k}}{K}\right)^{-\frac{1}{0.5}} \\
r_{k}^{(1)}=r_{k-1}^{(1)}+d_{k}^{(1)} \\
l_{k}^{(1)}=\left(l_{0}^{2}-\frac{\left.\ln \frac{r_{k}^{(1)}}{r_{0}}\left(l_{0}^{2}-l_{n}\right)\right)^{0.5}}{\ln \frac{r_{n}}{r_{0}}}\right. \\
\text { And } E_{r k}^{(1)}=\frac{U}{2} \frac{\left(l_{0}+l_{n}\right)}{\ln \frac{r_{n}}{r_{0}}} \frac{1}{r_{k}^{(1)} l_{k}^{(1)}}
\end{gathered}
$$

Then the voltage of layer $k$ can be obtained as follows:

$$
\Delta U_{k}^{(1)}=\frac{2 E_{r k}^{(1)} l_{k}^{(1)} r_{k}^{(1)} \ln \frac{r_{k}^{(1)}}{r_{k-1}^{(1)}}}{l_{k-1}^{(1)}+l_{k}^{(1)}}
$$

In this way, the length of the upper and lower steps of the bushing core can be determined:

$$
\lambda_{1 k}^{(1)}=\frac{\Delta U_{k}^{(1)}}{E_{a 1 k}} \quad \lambda_{2 k}^{(1)}=\frac{\Delta U_{k}^{(1)}}{E_{a 2 k}}
$$

On the basis of equal margin design, the design of capacitor core and plate can be carried out. In this design, a number of small plates are inserted between the main plates, and the edge breakdown of the core is avoided by using the strengthening effect of the thin dielectric layer. Generally, $X J_{l}$ small plates are inserted between the main plates of $D J_{1}$ layer near the center guide rod side, $X J_{3}$ small plates are inserted between the main plates of $D J_{3}$ layer near the flange side, and $X J_{2}$ small plates are inserted between the main plates of $D J_{2}$ layer in the middle of the core:

$$
N=D J_{1}+D J_{2}+D J_{3}
$$

The size and position of the $N$ main plates can be determined by the equal margin design method, and the small plates are designed according to the equal step and equal capacitance. Therefore, the starting voltage of partial discharge between main plates can be calculated by formula (15):

$$
U_{i i}=K\left(X J_{i}+1\right)\left(\frac{D_{k}}{X J_{i}+1}\right)^{0.5}(i=1,2,3)
$$

In the above formula, $K$ is the same as in formula (1), and $D_{k}$ is the thickness between main plates. According to the size of the outer contour of the core and the operating voltage, the respective number of the large and the small plates is determined. According to the equal margin design method, the thickness $d_{k}$ of the insulation layer between the plates when the total number of plates is calculated, where $n$ is calculated by formula (16):

$$
n=\sum_{i=1}^{3} D J_{i}\left(X J_{i}+1\right)
$$

Then, according to $d_{k}$, the thickness $D_{i}$ of the insulation layer between the main plates is determined. The calculation method is shown in equations (17)-(19), where $h$ is the thickness of the single layer corrugated paper:

$$
\begin{gathered}
D_{i}=\operatorname{int}\left(\frac{\sum_{k=1}^{D J_{1}\left(X J_{1}+1\right)} d_{k}}{D J_{1}\left(X J_{1}+1\right) h}+P I T_{5}\right) h\left(X J_{1}+1\right) \\
\left(i=1,2, \ldots, D J_{1}\right) \\
D_{i}=\operatorname{int}\left(\frac{\left.\sum_{k=D J_{1}\left(X J_{1}+1\right)+1}^{D J_{2}\left(X J_{2}+1\right) h}+P I T_{5}\right) h\left(X J_{2}+1\right)}{\left(i=D J_{1}+1, \ldots, D J_{1}+D J_{2}\right)}\right. \\
D_{i}=\operatorname{int}\left(\frac{k=D J_{1}\left(X J_{1}+1\right)+D J_{2}\left(X J_{2}+1\right)+1}{D J_{3}\left(X J_{3}+1\right) h}+P I T_{5}\right) h\left(X J_{3}+1\right) \\
\left(i=D J_{l}+D J_{2}+1, \ldots, N\right)
\end{gathered}
$$

In the above formula, $\operatorname{int}()$ is the rounding function, $P I T_{5}$ is the parameter introduced for rounding, and the value is 0.5 when rounding. After calculating the insulation layer thickness $D_{\mathrm{i}}$ between main plates, the radius $R_{\mathrm{i}}$ between main plates can be calculated by using formula (9), and then the length $L_{\mathrm{i}}$ of n-layer main plates can be determined according to formula (10). The voltage between plates, radial field strength and axial field strength of upper and lower parts can be calculated 
according to the above equal margin design, and the initial partial discharge voltage between main plates should be calculated according to formula (15).

\subsection{Software package implementation of the traditional bushing capacitor core equal margin design method}

The length and the position of the plates in each layer of the core can be determined by the above theoretical formula after finite cycle calculation, and the partial discharge margin and the axial field strength between the plates can be evenly distributed[12,13]. A software package is developed based on the equal margin design method for internal insulation of high voltage capacitive bushing. The interface of the software package is shown in Figure 2.
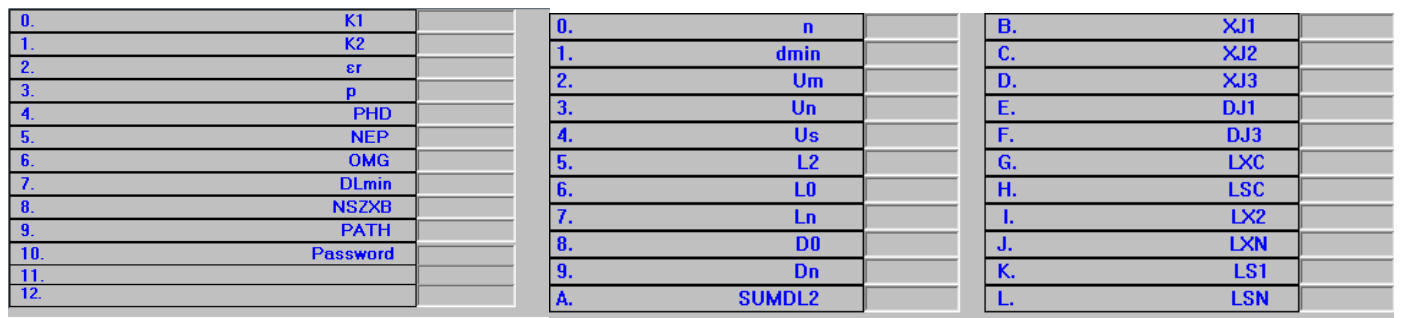

Fig.2 Packages for HV bushing condenser design

The existing core materials mainly include oil impregnated paper and epoxy impregnated paper. The material parameters need to be determined through specific tests, and the test voltage and design size parameters are determined according to the type of bushing. After the relevant parameters are set, the traditional equal capacitance method, equal thickness method, equal margin method and large and small plate method can be selected to design the structure parameters of bushing capacitor core. Through the above theoretical analysis, it can be seen that the premise of equal margin method to achieve equal partial discharge margin between plates and uniform distribution of axial field strength is that the electric field between plates of bushing core meets the theoretical calculation formula of coaxial cylindrical structure with linear medium. However, considering the model and temperature factors, the theoretical formula is not suitable for the calculation of the electric field between the plates of high voltage bushing. It is difficult to obtain ideal design results of the core by using the traditional high voltage capacitive bushing internal insulation optimization design software. It is necessary to further improve traditional high voltage bushing internal insulation optimization design program.

\section{Influence of high voltage bushing model and temperature on electric field distribution of core}

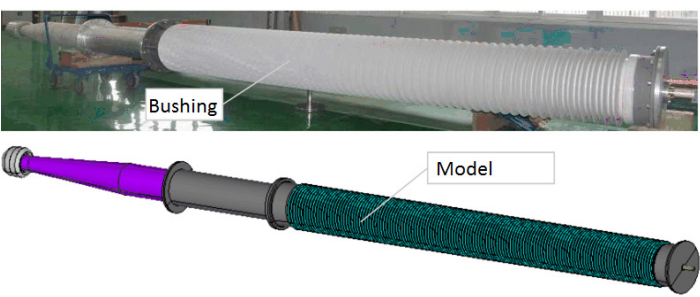

Fig.3 HV condenser bushing and FEM calculation model
In this paper, the large-scale finite element analysis is used as the analysis platform to further study the effect of the high-voltage bushing model and temperature factors on the core electric field distribution. Taking high voltage capacitive bushing as an example, the rated operating voltage and current of the bushing are $800 \mathrm{kV}$ and $3800 \mathrm{~A}$ respectively. The physical and finite element models of the bushing are shown in Fig. 3, and design parameters of the outer contour of the capacitor core are listed in Table 1.

Tab.1 Structure parameters of bushing condenser

\begin{tabular}{cccccc}
\hline $\begin{array}{c}\text { Structural } \\
\text { parameters }\end{array}$ & $L_{0}(\mathrm{~mm})$ & $L_{n}(\mathrm{~mm})$ & $D_{0}(\mathrm{~mm})$ & $D_{n}(\mathrm{~mm})$ & $n$ \\
\hline $\begin{array}{c}\text { Numerical } \\
\text { value }\end{array}$ & 9344 & 3346 & 135 & 526 & 93 \\
\hline
\end{tabular}

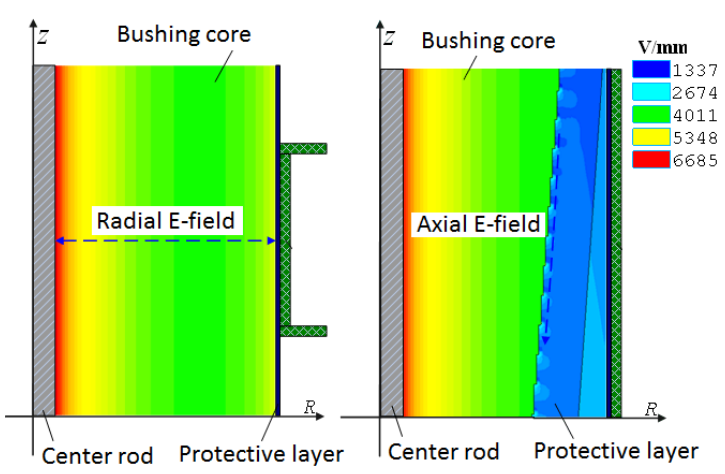

Fig.4 E-field distribution of bushing condenser with FEM

In Table $1, L_{0}, D_{0}$ are the length and diameter of the zero layer plate, $L_{n}, D_{n}$ are the length and diameter of the $n^{\text {th }}$ layer plate, and $n$ is the total number of plates in the bushing core. The above design parameters are input into the high voltage capacitive bushing internal insulation optimization design software package, and the large plate type and equal margin design methods are adopted. The radial field strength of the core under the highest operating voltage is $E_{r}$, the axial field strength under one 
minute power frequency withstand voltage is $E_{a}$, and the partial discharge margin between plates is $Y_{d m}$. In order to verify the correctness of the finite element calculation method in the electric field calculation of high voltage bushing capacitor core, the finite element calculation model of single capacitor core is established according to the structural parameters of the core output by the traditional bushing internal insulation optimization design software package calculation environment. The grid is divided and the electric potential load is applied for electric field simulation. The radial and axial electric field distribution is shown in Figure 4.

At the same time, the output field strength values of traditional optimization design software package for the bushing internal insulation are compared with the finite element calculation results in Fig.5, including the radial field strength of the core, the axial field strength and the partial discharge margin between the plates. The figure shows that the results of the software package are basically consistent with those of the finite element method, which proves the accuracy of the finite element method in calculating the internal electric field distribution of the bushing core. The results show that the radial electric field distribution of the bushing core presents U-shaped distribution of high at both ends and low in the middle, and the maximum electric field strength of the center guide rod side is higher than that of the flange side. The axial electric field strength of the core and the partial discharge margin between the plates fluctuate in a certain range, but the overall distribution tends to be uniform. The comparison results of the above core electric field distribution show that: 1) using the traditional optimization design software package to design the bushing core can basically meet the design concept of axial field strength and uniform distribution of the partial discharge margin between plates; 2) the software package compiled according to the theoretical formula can be developed, and the calculation results are accurate.
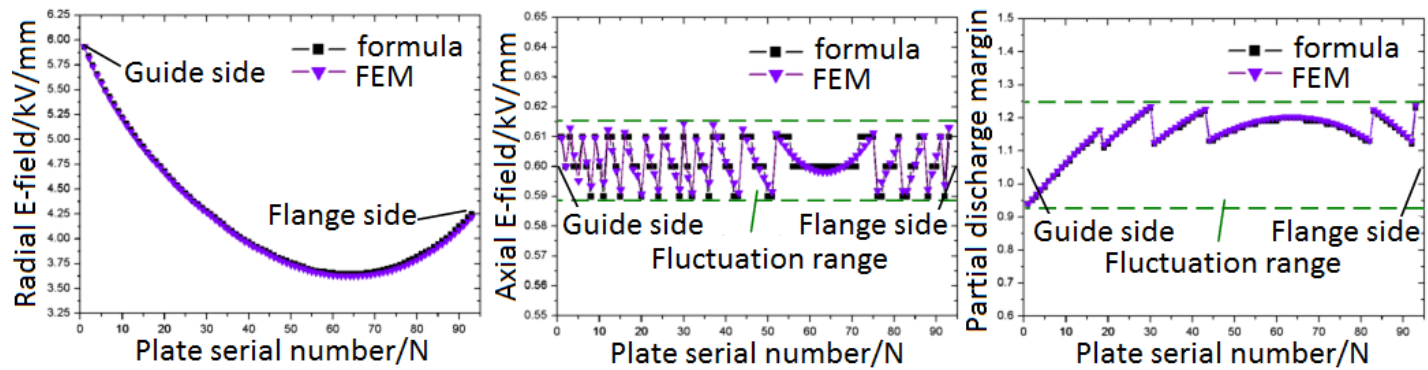

Fig.5 Comparison between packages and FEM

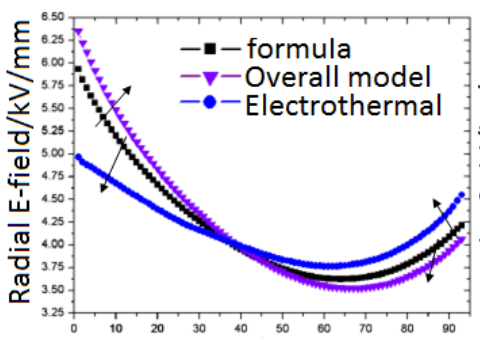

Plate serial number/N

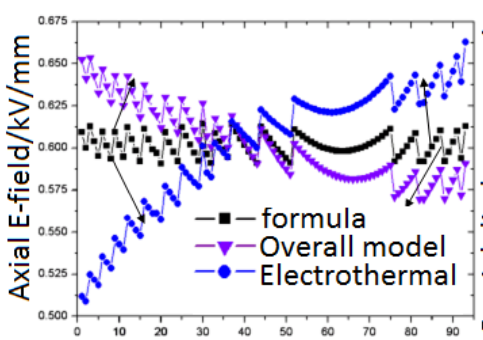

Plate serial number/N

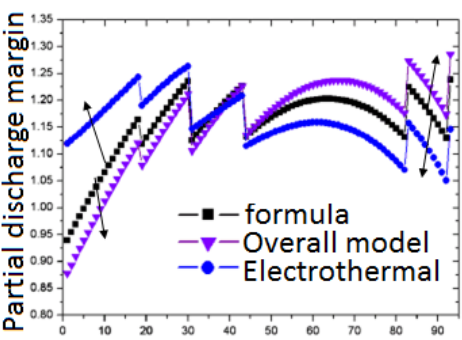

Plate serial number/N

Fig.6 Relationship between model and temperature factors with E-field inside bushing condenser

Compared with the traditional theoretical formula, the finite element method has more advantages: 1) the traditional theoretical formula only takes into account the influence of the main capacitance in the bushing core on the electric field distribution, which can not quantitatively express the distortion effect of stray capacitance on the electric field caused by the central guide rod, external flange, transformer tank and other metal parts. 2) the temperature gradient will appear in the core of high voltage bushing under the actual operation conditions of full voltage and full current. The distribution of electric field intensity will change obviously, and traditional theoretical formula can not describe it quantitatively. The effect of the above two factors on the electric field distribution of the bushing core is shown in Fig. 6. It can be seen that the model and temperature have significant effects on the internal electric field of the core: the model factor makes the radial electric field strength and axial electric field strength of the center guide rod side increase, the partial discharge margin value decrease, and the change trend of the three parameters of the flange side is opposite. The temperature factor makes the radial electric field strength and axial electric field strength of the center guide rod side increase when the electric field strength decreases, the partial discharge margin increases, and the change trend of the three parameters on flange side is opposite. Therefore, it is necessary to improve the traditional high voltage bushing insulation optimization design software package under the condition of finite element calculation, that is, to realize the uniform distribution of axial field strength and partial discharge margin of capacitor core when considering the model and temperature influence factors. 


\section{Application example of improved equal margin design method for internal insulation of high voltage bushing}

\subsection{Optimization design of structural parameters of UHV dry oil and gas bushing core}

The principle and algorithm flow of improved equal margin design method for the internal insulation of $\mathrm{HV}$ bushing were introduced in detail. The optimization design method is applied to the internal insulation design of UHV $1100 \mathrm{kV}$ epoxy impregnated dry Oil-SF6 bushing. The external outline of the initial design of the bushing is shown in Figure 7. The two ends of the core are respectively placed in the transformer oil tank and GIS pipeline gas chamber, and the central guide rod is connected. The electric field is shielded by the pressure equalizing cover. The length and diameter $L_{0}$ and $D_{0}$ of the zero layer plate are $4410 \mathrm{~mm}$ and $150 \mathrm{~mm}$ respectively, the length and radius $L_{n}, D_{n}$ of the $n^{\text {th }}$ layer plate are $1040 \mathrm{~mm}$ and $640 \mathrm{~mm}$ respectively, and the total number of plates $n$ in the bushing core is 95 . The rated working voltage of bushing is $635 \mathrm{kV}$ and the rated current carrying capacity is $3150 \mathrm{~A}$.

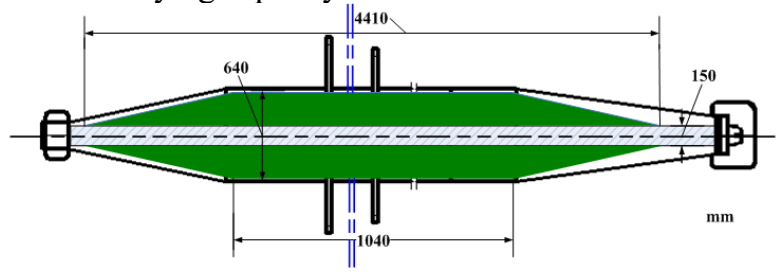

Fig.7 Outlet of UHV oil-gas bushings
In the calculation of temperature field, the Joule heating $P_{1}$ of core insulating medium and the eddy current heating $P_{2}$ of the central guide rod should be considered simultaneously, and the temperature boundary, convection and radiation conditions should be set[16].

$$
P_{1}=U^{2} 2 \pi f C \tan \delta
$$

In above formula, $U$ is the highest working phase voltage of the bushing, $f$ is the voltage frequency, $C$ is the main capacitance value of the bushing core, and $\tan \delta$ is the tangent value of the insulation dielectric loss angle of the core. The eddy current heating of the center guide rod is calculated by formula (21):

$$
P_{2}=I^{2} \operatorname{real}\left(\frac{\sqrt{i 2 \pi f \mu \gamma}}{2 a \pi \gamma} \frac{I_{0}(k a) K_{1}(k b)+K_{0}(k a) I_{1}(k b)}{I_{1}(k a) K_{1}(k b)-K_{1}(k a) I_{1}(k b)}\right)
$$

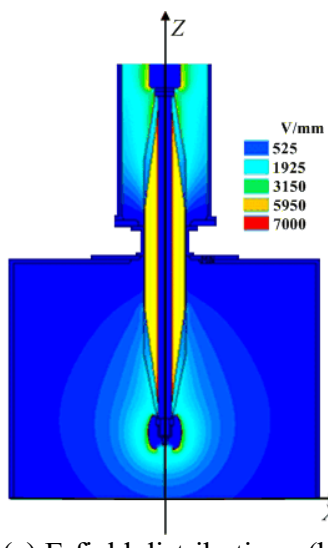

(a) E-field distribution (b) Temperature distribution

Fig.8 Temperature and E-field distribution under full model of UHV oil-gas bushings
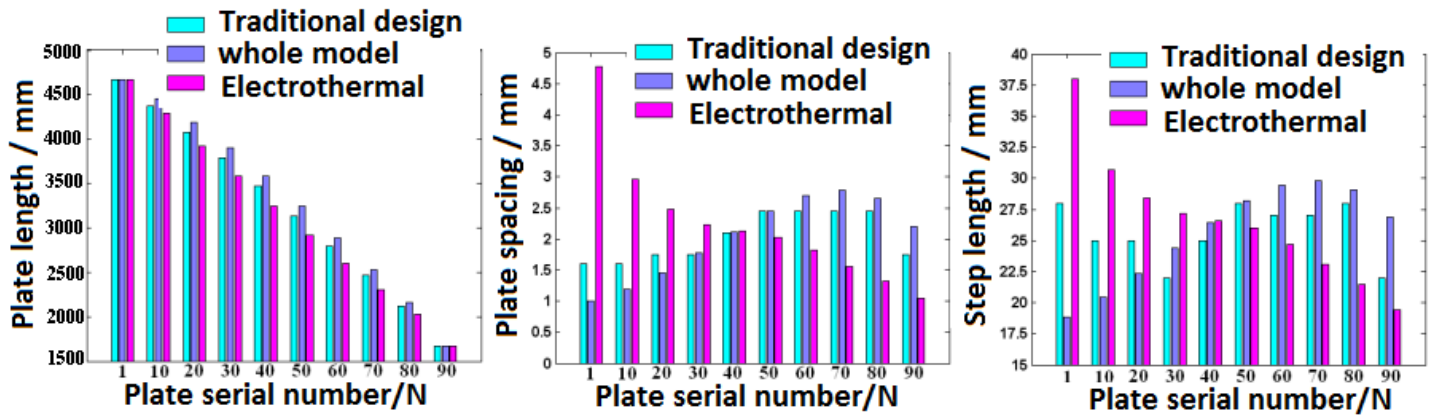

Fig.9 Optimized bushing condenser structure under different calculation conditions

In the above formula, real() is the real part function, $a, b$ are the outer radius and inner radius of the central guide rod, and $\gamma, \mu$ are the conductivity and permeability of the guide rod material, and $I_{i}, K_{i}(i=0,1)$ are the first and the second Bessel functions respectively. The full model electric field and temperature distribution of UHV oil and gas bushing in rated operation under calculation environment is shown in Figure 8.

Figure 9 shows that the high field strength area of bushing is mainly concentrated in the inner core and the surface of the pressure equalizing cover, and the hottest spot is about $95^{\circ} \mathrm{C}$, which is located near the interface between the center guide rod and the core. The optimization design of UHV oil and gas bushing core is carried out under the condition of full model and electric heating coupling calculation by using the method of equal margin design of high pressure bushing inner insulation improvement. The optimization results of plate length $A L_{i}$, plate spacing $D D_{i}$ and lower step length $S D L_{2 i}(i=1 \sim n)$ are compared as shown in Figure 10. 

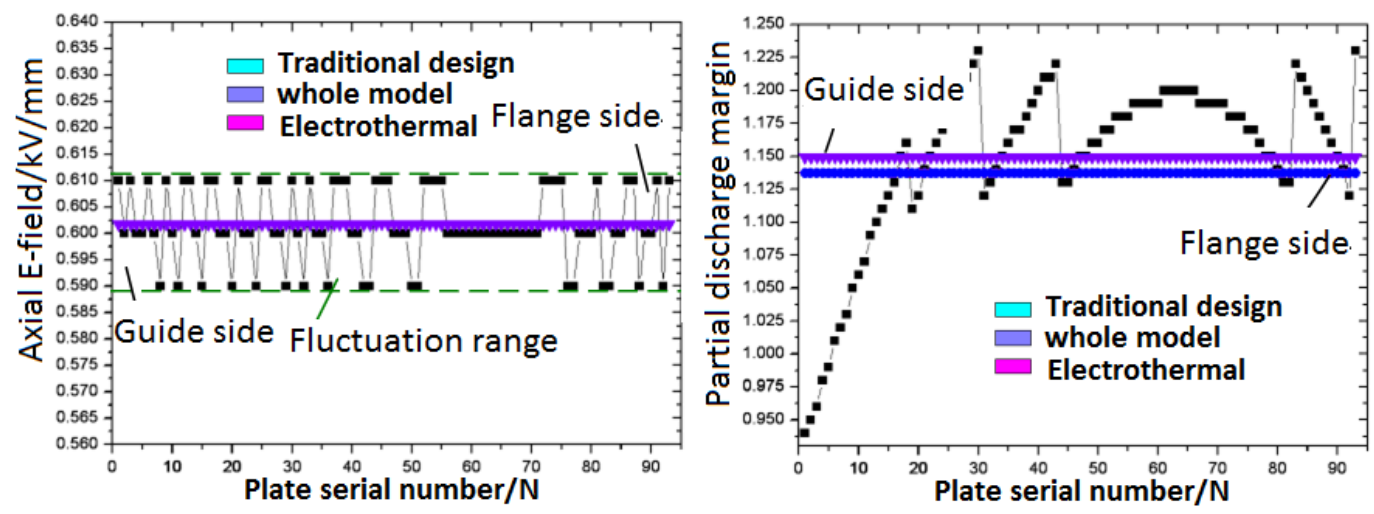

Fig.10 Optimized electric parameters results under different calculation conditions

Figure 9 shows that under the three calculation environments of traditional design, full model and electro thermal coupling, the optimized structural parameters obtained by using the improved internal insulation margin design method of high voltage bushing have obvious differences. In the three cases mentioned above, the length of the plate $\mathrm{AL}_{\mathrm{i}}$ decreases from the innermost layer (center guide rod side) to the outermost layer (flange side), the length of the plate is the longest in the full model condition, the shortest in the electrothermal coupling condition, and the middle in the traditional design condition; the plate spacing $\mathrm{DD}_{\mathrm{i}}$ in the traditional design and full model design conditions, the thickest dielectric layer is located in the middle of the core, but in the electrothermal coupling condition, The results show that the distance between plates decreases gradually, that is, the distance near the center guide rod is the largest, and the distance near the flange is the smallest. Figure 10 shows the comparison between the optimized electric field parameters of the bushing core under the condition of full model and electrothermal coupling and the traditional design. It can be seen that under the traditional design, the axial field strength of the bushing core fluctuates in the strip area, and the partial discharge margin fluctuates greatly. The minimum value of partial discharge margin between the innermost plates (center guide rod side) is 0.925 . Because the initial partial discharge voltage of the whole bushing core is determined by the minimum value of partial discharge margin between the plates, the traditional design is not conducive to the long-term safe and stable operation of the bushing core. By using the improved equal margin method, the axial field strength of the bushing core and the partial discharge margin between the plates can be evenly distributed. Under the condition of the whole model, the PD margin between plates is 1.155 . Theoretically, the initial PD voltage of the whole core is increased from $587 \mathrm{kV}$ to $733 \mathrm{kV}$, and the axial field intensity is evenly distributed, which reduces the possibility of flashover at the end of the core. Under the condition of power frequency AC, the influence of model factors on the core electric field distribution is greater than that of temperature factors. Therefore, the core structure parameters optimized under the full model conditions are used for rolling, pouring and curing. The prototype is shown in Figure 11.

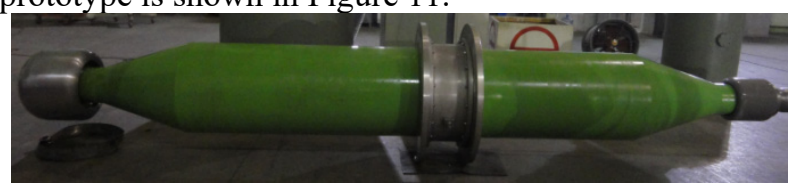

Fig.11 Prototype of UHV oil-gas bushings

\section{Conclusion}

In this paper, an improved design method of the equal margin for internal insulation of high-voltage capacitive bushing is realized by combining electrothermal coupling calculation and genetic algorithm in finite element calculation environment, and a prototype of UHV oil and gas bushing is successfully developed by using this method:

1) Considering the structure and isothermal condition of the bushing capacitance core, the electric field distribution inside the core calculated by the traditional design software package is consistent with that calculated by the finite element method, which proves the effectiveness of the finite element method in calculating the electric field distribution inside the bushing core.

2) The model and temperature factors have a significant influence on the electric field distribution in the bushing core. In the finite element calculation environment, electrothermal coupling calculation method can be used to quantitatively describe the influence law. On this basis, the traditional equal margin design method is improved to realize the uniform distribution of axial field strength of bushing core and partial discharge margin between plates.

3) The improved equal margin design method is applied to the core structure optimization design of UHV oil and gas bushing prototype. The developed prototype has passed all type tests, which proves the effectiveness and rationality of the improved equal margin design method.

4) The improved equal margin design method proposed in this paper has certain theoretical guidance value for other types of UHV AC/DC bushing core design. 


\section{References}

1. LIU Zhen-ya, Ultra-high voltage power grid[M], Beijing, China: China Economy Press, 2005.

2. Zhu Fang, Zhao Ziyu, Liu Qichang, Ju Jichun. A New Design Method for Condenser Body of H.V. Transformer Bushing[J]. Journal of Xi'an Jiaotong University, 1989, 23(1): 77-100.

3. Xie Heng-kun. Electrical insulation design principles [M]. Beijing, China: Mechanical Industry Press, 1992.

4. N.S.Jyothi, T.S.Ramu, Manoj Mandlik. Temperature distribution in resin impregnated paper insulation for transformer bushing[J]. IEEE Transaction on Dielectrics and Electrical Insulation, 2010, 17(3): 931-938.

5. M.Clemens, E.Gjonaj, P.Pinder, T.Weiland. Numerical simulation of coupled transient thermal and electromagnetic fields with the finite integration method[J]. IEEE Transactions on Magnetics, 2000, 36(4): 1448-1452.

6. Daniel Weida, Thorsten Steinmetz, Markus Clemens. Electro-quasistatic high voltage field simulations of large scale insulator structures including 2D models for nonlinear field-grading material layers[J]. IEEE Transactions on Magnetics, 2009, 45(3): 980-983.

7. Han S.J., Zou J., Gu S. Q et al. Calculation of the potential distribution of high voltage metal oxide arrester by using an improved semi-analytic finite element method[J]. IEEE Transactions on Magnetics, 2005, 41(5): 1392-1395.

8. Zheng Libing, Han Li, Liu Jun, Wen Xuhui. Investigation of the temperature character of IGBT failure mode based on 3D thermal-electro coupling FEM[J]. Transaction of China Electrotechnical Society, 2011, 26(7): 242-246.
9. Su Xiuping, Lu Jianguo, Liu Guojin. Thermal field simulation analysis of miniature DC electromagnetic relays[J]. Transactions of China Electrotechnical Society, 2011, 26(8): 185-189.

10. Ch.Chakradhar Reddy, T.S.Ramu. Estimation of thermal breakdown voltage of HVDC cables-A theoretical framework[J].IEEE Transaction on Dielectrics and Electrical Insulation, 2007, 14(2): 400-408.

11. Du Canxun, Gui Weihua, Zhou Guanghou. Excessive losses and temperature field calculation of stator coil bars in air-cooled large hydrogenerators[J]. Proceedings of the CSEE, 2012, 32(12): 111-119.

12. Dang Sha-sha, $\mathrm{Xu}$ Yang, Zhang Hong-song. ANSYS12.0 finite element analysis of multiphysics coupling field from start to master [M]. Beijing, China: China Mechanical Press, 2010.

13. Ding Shuye, Ge Yunzhong, Sun Zhaoqiong et al. Calculation and analysis of fluid field and temperature field for high-altitude type doubly-fed wind generators $[\mathrm{J}]$. Proceedings of the CSEE, 2012, 32(24): 74-79.

14. Li Weili, Chen Yuhong, Huo Feiyang et al. Fluid flow and temperature field analysis between two poles of a large air-cooled hydro-generator rotor in rotation[J]. Proceedings of the CSEE, 2012, 32(9): 132-139.

15. Zhang Xiaochen, Li Weili, Qiu Hongbo et al. Calculation of Electromagnetic Field and Temperature Field in Super High Speed Permanent Magnet Generator With Composite Structures[J]. Proceedings of the CSEE, 2011, 31(30): 85-92.

16. Lu Xiaolong, Zhang Jianhui, Zhao Chunsheng. Temperature Field Analysis for Traveling-wave Rotary Ultrasonic Motor[J]. Proceedings of the CSEE, 2011, 31(18): 33-39. 